\title{
Squamous cell carcinoma arising in plaque-like oral lichen planus: A case report
}

\section{Powstanie raka płaskonabłonkowego w przebiegu płytkopodobnej postaci liszaja płaskiego w jamie ustnej - opis przypadku}

\author{
Chanwit Prapinjumrune ${ }^{1, A-F}$, Risa Chaisuparat ${ }^{2, D-F}$, Thongprasom Kobkan ${ }^{1, A-F}$ \\ 1 Oral Medicine Department, Faculty of Dentistry, Chulalongkorn University, Bangkok, Thailand \\ 2 Oral Pathology Department, Faculty of Dentistry, Chulalongkorn University, Bangkok, Thailand \\ A - research concept and design; $\mathrm{B}$ - collection and/or assembly of data; $\mathrm{C}$ - data analysis and interpretation; \\ $D$ - writing the article; $E$ - critical revision of the article; $F$ - final approval of article
}

Address for correspondence

\section{Thongprasom Kobkan}

E-mail: kobkan.t@chula.ac.th

Funding sources

none declared

Conflict of interest

none declared

\section{Acknowledgements}

We express our sincere thanks to professor dr. Somporn Swasdison and assistant professor dr. Ekarat Phattarataratip, Oral Pathology Department, Faculty of Dentistry, Chulalongkorn University, for their histopathological reports. We thank dr. Kevin Tompkins for editing the manuscript and the Oral Medicine Department staff for their assistance.

Received on February 16, 2017

Revised on April 18, 2017

Accepted on April 19, 2017

\begin{abstract}
Oral lichen planus (OLP) is a chronic inflammatory disease of the oral mucosa that commonly occurs in middle-aged or elderly women.

In this paper, we describe a case of a 64-year-old woman who presented with red and white lesions in the oral cavity. Intraoral examination revealed a white plaque and white lines with a mild erythematous area on the right buccal mucosa, however, the left buccal mucosa showed only white striae and a mild erythematous area. Biopsy specimens taken twice from the right buccal mucosa demonstrated OLP characteristics. After 7-years of follow-up, an irregularly shaped ulcer was observed on the plaque lesion of the right buccal mucosa. A biopsy was performed again and the final diagnosis of superficially invasive squamous cell carcinoma was made. OLP is classified as a potentially malignant disorder (PMD) of the oral mucosa. Histopathological examination is mandatory to confirm the diagnosis and to exclude dysplasia and malignancy. Due to the malignant potential of OLP, long-term follow-up is strongly recommended, particularly in elderly patients with plaque-like OLP.
\end{abstract}

Key words: the elderly, lichen planus, squamous cell carcinoma, potentially malignant disorders Słowa kluczowe: osoby starsze, liszaj płaski, rak płaskonabłonkowy, zmiany przednowotworowe
DOI

$10.17219 / \mathrm{dmp} / 70528$

Copyright

○ 2017 by Wroclaw Medical University

and Polish Dental Society

This is an article distributed under the terms of the

Creative Commons Attribution Non-Commercial License

(http://creativecommons.org/licenses/by-nc-nd/4.0/) 
Lichen planus (LP) is a chronic mucocutaneous disease that was first described and named by Sir Erasmus Wilson in $1869 .^{1}$ This disease may occur on the skin and/or oral mucosa. Oral lichen planus (OLP) is a common inflammatory disease of the oral mucosa that involves cell-mediated immunological dysfunction. ${ }^{2,3}$ The prevalence of OLP has been reported as $1.3 \%$ and it predominantly presents in middle-aged or elderly women. ${ }^{4}$ OLP has various clinical manifestations, such as reticular, papular, plaque, atrophic, and ulcerative (erosive) patterns. ${ }^{2}$ OLP lesions present bilaterally and are most commonly found on the buccal mucosa. However, OLP can appear on the tongue, gingiva, and mucobuccal fold. Recently, OLP was classified as a potentially malignant disorder (PMD) of the oral mucosa. ${ }^{5}$ The malignant potential of OLP remains a point of debate in the literature. However, it is important to closely follow up a patient diagnosed with OLP. ${ }^{6}$ Therefore, histopathological examination by expert oral pathologists is mandatory to confirm the clinical diagnosis and to exclude dysplasia and malignant transformation. ${ }^{7,8}$

\section{Case report}

A 58-year-old female first presented at the Oral Medicine clinic, Faculty of Dentistry, Chulalongkorn University in 2008 with bilateral lesions on the buccal mucosa and gingiva without skin lesions. The patient's history revealed no significant medical problems. Moreover, this patient reported no history of allergy, alcohol consumption, or tobacco smoking; and was not taking any medications.

At the patient's first visit, intraoral examination revealed white striae with mild erythematous areas on the bilateral buccal mucosa. There were no obviously contributing factors causing chronic irritation in the lesional areas and she had no history of denture wearing. Based on the patient's history and clinical examination, our initial clinical impression was atrophic OLP. However, the patient refused histopathological examination of her lesions. The patient was advised to apply triamcinolone acetonide in orabase (TAO) $0.1 \% 3$ times a day. In addition, we also educated the patient on how to maintain good oral hygiene. At the 1 month follow-up, the lesions showed significant improvement.

After discontinuing follow-up visits for more than 6 years, the patient returned to our clinic in 2014 with a chief complaint of a burning sensation in the oral cavity. A white plaque and mild white striae with a mild erythematous area were observed on the right buccal mucosa (Fig. 1A). Mild erythematous areas with faint ill-defined keratotic lines were seen on the left buccal mucosa and gingiva. Furthermore, hyperpigmentation was also observed on the left buccal mucosa (Fig. 1B). At this visit, an incisional biopsy was performed on the lesion on the right buccal mucosa. The histopathological features of the biopsied tissue were suggestive of OLP (Fig. 1C); however,

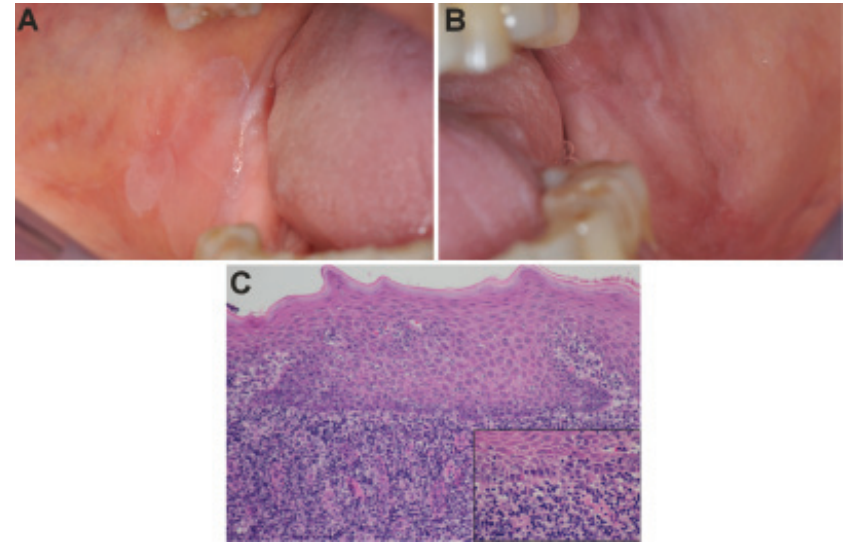

Fig. 1. Patient's initial presentation. A) Clinical presentation of the white plaque and white lines with a mild erythemathous area on the right buccal mucosa. B) White striae and erythematous area with hyperpigmentation on the left buccal mucosa. C) Biopsy specimen from the right buccal mucosa showed hyperkeratinizing stratified squamous epithelium with irregular rete ridges. A disrupted basilar area is seen in the inset. The connective tissue is notable for a band-like infiltrate of lymphocytes (hematoxylin-eosin stain, original magnification $\times 10$, inset $\times 40$ )

the direct immunofluorescence (DIF) results showed negative findings. Moreover, her serum antinuclear antibody (ANA) was positive at a low titer $(1: 80)$ with speckled and nucleolar patterns. We prescribed TAO $0.1 \%$ to be applied to the lesions 3 times a day and recalled the patient for evaluation every 3 months, however, the patient did not come to the clinic during the time of the follow-up period because she had no compliant.

At a follow-up 11 months after the biopsy was taken and applying TAO $0.1 \%$, the lesions on the right buccal mucosa presented as a thick white plaque and white lines with a mild erythematous area (Fig. 2A), however, only mild white striae and an erythematous area on the left buccal mucosa were seen (Fig. 2B). A biopsy was performed again on the plaque lesion of the right buccal mucosa. The histopathological findings confirmed the prior diagnosis of OLP (Fig. 2C). However, the DIF and serum ANA assay findings were negative. We prescribed TAO $0.1 \%$ to be applied to the lesions 3 times a day and recalled the patient for evaluation every 3 months. Interestingly, during the follow-up period, the lesions showed improvement and the patient was asymptomatic. Although the lesions were asymptomatic and without erosion, those lesions showed mild erythematous areas. Thus, we advised the patient to stop using the topical steroid and to apply protein-free hemodialysate $5 \%$ and polidocanol $1 \%$ twice a day instead of the treatment of mild oral mucosal inflammation.

Fifteen months after the second biopsy, an irregularly shaped ulcer was present on top of the well-defined margin of the white plaque on the right buccal mucosa (Fig. 3A). Reticular white striae with a mildly erythematous area were clearly observed on the left buccal mucosa (Fig. 3B). An incisional biopsy was performed again on the plaque lesion of the right buccal mucosa and microscopic examination revealed several nests and islands of malignant epithelial 

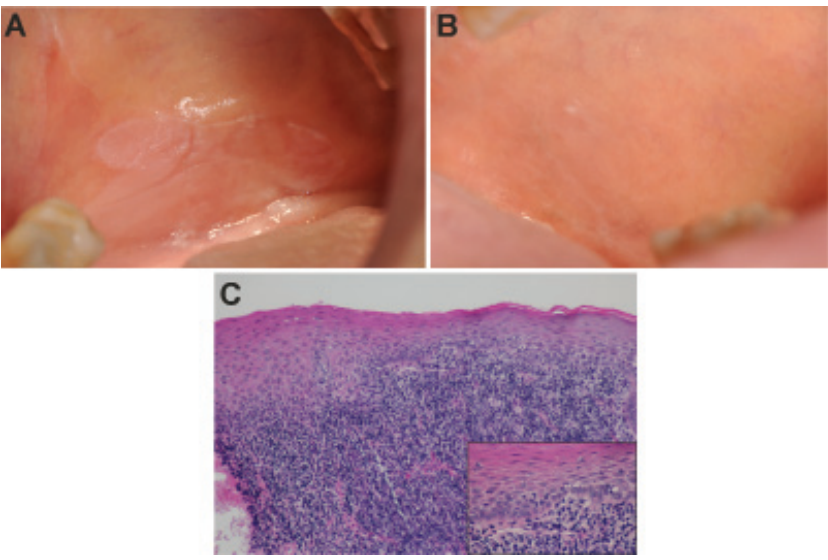

Fig. 2. The relapse of the lesion at 11 months follow-up. A) Clinical presentation of a thick white plaque and white lines with a mild erythematous area on the right buccal mucosa. B) Mild white striae and an erythematous area on the left buccal mucosa. C) Biopsy specimen from the right buccal mucosa displayed hyperplastic keratotic squamous epithelium. Degeneration and infiltration of lymphocytes into the basal cell layer are observed in the inset. The underlying connective tissue contains a band of lymphocytes (hematoxylin-eosin stain, original magnification $\times 10$, inset $\times 40$ )

cells invading to the superficial underlying stroma. The neoplastic epithelial cells exhibited cellular pleomorphism, prominent nucleoli and increased mitotic activity. An inflammatory reaction was noted (Fig. 3C). The final diagnosis was superficially invasive squamous cell carcinoma. We counseled the patient about the diagnosis and immediately referred the patient to the cancer center at Chulalongkorn hospital for further evaluation and treatment.

\section{Discussion}

OLP is a chronic inflammatory disease that can reduce patient's quality of life. Numerous studies have reported that immunologic dysfunction is a crucial factor involved in the pathogenesis of OLP. ${ }^{2,3,9}$ The altered immune function causes a cytotoxic $\mathrm{T}$ cell attack on the basal keratinocytes that presents with various clinical appearances. Clinically, OLP presents as reticular, papular, plaque, atrophic, and ulcerative/erosive types. ${ }^{2}$ The most common of these types is reticular lesions that are typically asymptomatic.? Normally, reticular OLP presents as prominent white striae, however, faint white lines may also be present. The atrophic and ulcerative/erosive types of OLP can cause a burning sensation and pain when patients eat hot and spicy food. ${ }^{8}$ Although plaque-type OLP is found less frequently, this type of OLP requires close attention to carefully distinguish plaque-type OLP from leukoplakia.

Although previous studies have reported variability in the histopathologic assessment of OLP among oral pathologists, histopathological evaluation is mandatory to confirm the clinical diagnosis.,10 Moreover, additional methods, such as DIF evaluation and assaying serum ANA, are suggested to be performed to accurately diagnose OLP. DIF is widely used to detect OLP, especially in

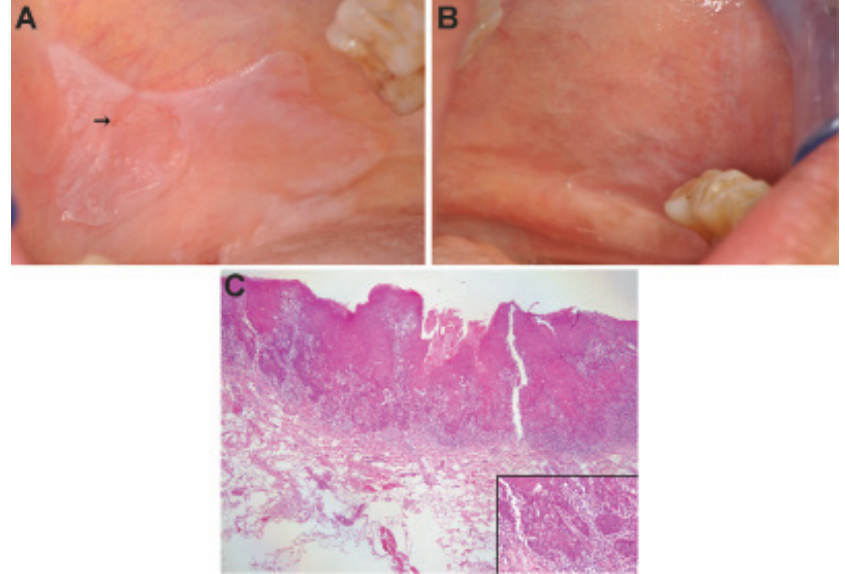

Fig. 3. Fifteen months follow-up. A) The clinical presentation of a thick white plaque with a small irregular shape ulcer on the right buccal mucosa. B) Reticular white striae on the left buccal mucosa with an erythematous area. C) Microscopic examination from the right buccal mucosa revealed parakeratinized stratified squamous epithelium with areas of dysplasia. The superficial underlying stroma showed several nests and islands of malignant epithelial cells. The neoplastic cells exhibit cellular pleomorphism, prominent nucleoli, and increased mitotic activity is seen in the inset. (hematoxylin-eosin stain, original magnification $\times 4$, inset $\times 20$ )

cases where the histopathological features do not allow for a definitive diagnosis. ${ }^{7}$ This method has proved to be a valuable tool in the diagnosis of a number of oral mucosal diseases and remains the gold standard for the differential diagnosis from other lesions such as lupus erythematosus (LE), pemphigus vulgaris, mucous membrane pemphigoid (MMP), and chronic ulcerative stomatitis (CUS). ${ }^{11-13}$ In general, DIF studies of OLP show positive findings of shaggy fibrin deposition at the basement membrane zone with or without IgM cytoid bodies. ${ }^{11,12,14,15}$ In our patient, although the results of the DIF examination were negative, the clinical appearance of the OLP lesions present on the buccal mucosa bilaterally and the histopathological reports of the biopsy specimens taken twice from the most representative area at the same spot were suggestive of OLP without epithelial dysplasia. In addition, this patient did not take any medications and did not have any contacting metal material close to the lesions. Therefore, oral lichenoid lesions (OLLs) were excluded. The diagnosis of the lesions in this patient was made based on the modified criteria of van der Meij et al. ${ }^{16}$ Moreover, the lesions in this case showed no signs of oral candidiasis and there was no presence of any contributing factors causing Candida infection in this patient. Finally, the definite diagnosis of her oral lesions from 2 biopsies together with the oral manifestations confirmed OLP.

Interestingly, a recent report showed that OLP-like lesions in elderly Thais positive for ANA could be diagnosed as having CUS-like lesions or OLP, however, LE could not be entirely ruled out. ${ }^{17}$ Indeed, in our experience, red and white lesions that are similar to OLP lesions could be manifestations of other uncommon oral diseases. Therefore, lesions with the characteristics, as reported by oral pathologists, compatible with a diagnosis of OLP 
and a DIF evaluation with negative findings should be paid careful attention.

Assaying serum ANA is a useful diagnostic method for screening for autoimmune diseases. Our recent study found that positive serum ANA was detected in $73.9 \%$ of Thai OLP patients. ${ }^{18}$ However, there was no relationship between the serum ANA titer level and the clinical features in the present case. After close follow-up of the present case for 15 months, the plaque-like OLP on the right buccal mucosa transformed into superficially invasive squamous cell carcinoma.

OLP is a common chronic disease affecting patient's quality-of-life. Treatment of chronic diseases usually takes a long time and requires long-term follow-up. Hence, recall and evaluation of OLP patients is crucial for assessing the response of the lesions to treatment. Although there is no current consensus on a specific time frame for OLP and OLL follow-up, we recalled this patient to evaluate the oral lesion every 3 months. However, the patient was not cooperative in coming for regular follow-up visits and returned only when she needed to. She had discontinued recall visits for 6 years after treating her the first time and for 15 months the second time. Finally, she came back for oral examination with the chief complaint of an unhealing ulceration on the right buccal mucosa. Unfortunately, histopathological findings indicated the development of early stage carcinoma.

OLP is currently classified as a PMD. ${ }^{5}$ Numerous studies on the development of squamous cell carcinoma in OLP patients have been reported. These studies, observing OLP lesions for 3 months-24 years, found malignant transformation of OLP ranging from $0-12.5 \%{ }^{6}$ Although the pathogenesis of the malignant transformation of OLP has not been clarified, a correlation between transformation and immunosuppressive agents, such as tacrolimus, which are used in treating OLP, has been reported. ${ }^{19,20}$ To our knowledge, the OLP lesions in this patient were treated with TAO $0.1 \%$, however, there are no reports about the relationship between treating OLP with TAO $0.1 \%$ and malignant transformation.

In the present case, we documented the development of superficially invasive squamous cell carcinoma in a lesion previously diagnosed as plaque-like OLP at the seventh year of follow-up. This report demonstrates the possible malignant potential of OLP. Close follow-up is strongly recommended for OLP patients; particularly elderly patients with plaque-like OLP that greatly resemble leukoplakia.

\section{References}

1. Farhi D, Dupin N. Pathophysiology, etiologic factors, and clinical management of oral lichen planus, part I: Facts and controversies. Clin Dermatol. 2010;28,100-108.

2. Lodi G, Scully C, Carrozzo M, Griffiths M, Sugerman PB, Thongprasom K. Current controversies in oral lichen planus: Report of an international consensus meeting. Part 1. Viral infections and etiopathogenesis. Oral Surg Oral Med Oral Pathol Oral Radiol Endod. 2005;100:40-51.
3. Thornhill MH. Immune mechanisms in oral lichen planus. Acta Odontol Scand. 2001;59:174-177.

4. McCartan BE, Healy CM. The reported prevalence of oral lichen planus: A review and critique. J Oral Pathol Med. 2008;37:447-453.

5. Warnakulasuriya S, Johnson NW, van der Waal I. Nomenclature and classification of potentially malignant disorders of the oral mucosa. J Oral Pathol Med. 2007;36:575-580.

6. Gonzalez-Moles MA, Scully C, Gil-Montoya JA. Oral lichen planus: Controversies surrounding malignant transformation. Oral Dis. 2008;14:229-243.

7. Eisen D, Carrozzo M, Bagan Sebastian JV, Thongprasom K. Number $\checkmark$ Oral lichen planus: Clinical features and management. Oral Dis. 2005;11:338-349.

8. Scully C, Carrozzo M. Oral mucosal disease. Lichen planus. Br J Oral Maxillofac Surg. 2008;46:15-21.

9. Sugerman PB, Savage NW, Walsh LJ, et al. The pathogenesis of oral lichen planus. Crit Rev Oral Biol Med. 2002;13:350-365.

10. van der Meij EH, Reibel J, Slootweg PJ, van der Wal JE, de Jong WF, van der Waal I. Interobserver and intraobserver variability in the histologic assessment of oral lichen planus. J Oral Pathol Med. 1999;28:274-277.

11. Laskaris G, Sklavounou A, Angelopoulos A. Direct immunofluorescence in oral lichen planus. Oral Surg Oral Med Oral Pathol. 1982;53:483-487.

12. Firth NA, Rich AM, Radden BG, Reade PC. Assessment of the value of immunofluorescence microscopy in the diagnosis of oral mucosal lichen planus. J Oral Pathol Med. 1990;19:295-297.

13. Qari H, Villasante C, Richert J, Rees T, Kessler H. The diagnostic challenges of Separating chronic ulcerative stomatitis from oral lichen planus. Oral Surg Oral Med Oral Pathol Oral Radiol. 2015;120:622-627.

14. Helander SD, Rogers RS. The sensitivity and specificity of direct immunofluorescence testing in disorders of mucous membranes. J Am Acad Dermatol. 1994;30:65-75.

15. Kulthanan K, Jiamton S, Varothai S, Pinkaew S, Sutthipinittharm P. Direct immunofluorescence study in patients with lichen planus. Int J Dermatol. 2007;46:1237-1241.

16. van der Meij EH, van der Waal I. Lack of clinicopathologic correlation in the diagnosis of oral lichen planus based on the presently available diagnostic criteria and suggestions for modifications. J Oral Pathol Med. 2003;32:507-512.

17. Thongprasom K, Prapinjumrune $C$, Kanjanabuch P, Youngnak-Piboonratanakit $\mathrm{P}$, Preuksrisakul T. Correlation of serum ANA and direct immunofluorescence studies in elderly Thai patients with red and white oral lesions. J Oral Pathol Med. 2016;45:797-802.

18. Prucktrakul C, Youngnak-Piboonratanakit $P$, Kanjanabuch $P$, Prueksrisakul T, Thongprasom K. Oral lichenoid lesions and serum antinuclear antibodies in Thai patients. J Oral Pathol Med. 2015;44:468-474.

19. Morita M, Asoda S, Tsunoda K, et al. The onset risk of carcinoma in patients continuing tacrolimus topical treatment for oral lichen planus: A case report. Odontology. 2017;105:262-266.

20. Mattsson U, Magnusson B, Jontell M. Squamous cell carcinoma in a patient with oral lichen planus treated with topical application of tacrolimus. Oral Surg Oral Med Oral Pathol Oral Radiol Endod. 2010;110:19-25. 